\title{
Optical monitoring of glutamate release at multiple synapses in situ detects changes following LTP induction
}

\author{
Olga Kopach, Kaiyu Zheng and Dmitri A. Rusakov
}

\begin{abstract}
Information processing and memory formation in the brain relies on release of the main excitatory neurotransmitter glutamate from presynaptic axonal specialisations. The classical Hebbian paradigm of synaptic memory, long-term potentiation (LTP) of transmission, has been widely associated with an increase in the postsynaptic receptor current. Whether and to what degree LTP induction also enhances presynaptic glutamate release has been the subject of debate. Here, we took advantage of the recently developed genetically encoded optical sensors of glutamate (iGluSnFR) to monitor its release at CA3-CA1 synapses in acute hippocampal slices, before and after the induction of LTP. We attempted to trace release events at multiple synapses simultaneously, by using two-photon excitation imaging in fast frame-scanning mode. We thus detected a significant increase in the average iGluSnFR signal during potentiation, which lasted for up to $90 \mathrm{~min}$. This increase may reflect an increased amount of released glutamate or, alternatively, reduced glutamate binding to high-affinity glutamate transporters that compete with iGluSnFR.
\end{abstract}

Keywords: Glutamate release, Optical glutamate sensor, LTP, Two-photon excitation imaging, Acute hippocampal slices

\section{Introduction}

Hebbian postulates, which rationalise the principles of memory formation in the brain [1], have found their first experimental verification in the long-term potentiation (LTP) of excitatory transmission [2,3]. The majority of excitatory synapses in the cortex operate by releasing glutamate from presynaptic axons, the process that underpins rapid information processing and storage by neural circuits. Following decades of debate, it has been argued that the prevailing cellular mechanism underlying LTP rests with an increased current through postsynaptic receptors [4]. Experimental evidence for the alternative hypothesis, such as an increase in glutamate release probability [5-7], has been countered by an elegant hypothesis of silent synapses $[8,9]$ and by documenting no

\footnotetext{
* Correspondence: d.rusakov@ucl.ac.uk

Queen Square Institute of Neurology, University College London, Queen Square, London WC1N 3BG, UK
}

(c) The Author(s). 2020, corrected publication 2020. Open Access This article is licensed under a Creative Commons Attribution 4.0 International License, which permits use, sharing, adaptation, distribution and reproduction in any medium or format, as long as you give appropriate credit to the original author(s) and the source, provide a link to the Creative Commons licence, and indicate if changes were made. The images or other third party material in this article are included in the article's Creative Commons licence, unless indicated otherwise in a credit line to the material. If material is not included in the article's Creative Commons licence and your intended use is not permitted by statutory regulation or exceeds the permitted use, you will need to obtain permission directly from the copyright holder. To view a copy of this licence, visit http://creativecommons.org/ licenses/by/4.0/. The Creative Commons Public Domain Dedication waiver (http://creativecommons.org/publicdomain/zero/1. 0/) applies to the data made available in this article, unless otherwise stated in a credit line to the data.

increases in astroglial glutamate uptake post-induction $[10,11]$. However, the LTP-associated boost of release probability at non-silent synapses has subsequently been reported $[12,13]$ whereas no change in overall glutamate release can reflect hetero-synaptic depression at nonactive connections [14] or, more generally, rapid (pre) synaptic scaling $[15,16]$. The uncertainty has remained, largely because documenting glutamate release at individual synapses has had to rely on its physiological consequences rather than on release readout per se.

The advent of FM dyes decades ago was an important step in providing optical tools to detect exocytosis of synaptic vesicles $[17,18]$. More recently, the emergence of genetically encoded optical sensors for glutamate [19] has finally enabled direct monitoring of its release at individual synaptic connections. We showed earlier that, in certain imaging conditions, fluorescent glutamate 'sniffers' of the iGluSnFR family provide robust readout 
of glutamate release at identified synapses in organised brain tissue [20, 21], including in vivo [22]. In the present study, we take advantage of this approach in an attempt to understand changes in glutamate release properties at hippocampal Schaffer collateral axons, under the classical protocol of LTP induced by highfrequency afferent stimulation. We monitor LTP induction in the bulk of tissue, and analyse optical glutamate signals in arbitrary samples of presynaptic axonal boutons, which may correspond to both potentiated and non-potentiated synapses. In these settings, we aim to assess changes in glutamate release at individual synapses, and in the bulk of synaptic population.

\section{Results}

Viral delivery of iGluSnFR in neonates for multi-synapse glutamate imaging in situ

In our previous studies, we introduced optical glutamate sensors in the hippocampal neuropil via stereotaxic viral delivery in young animals [20] or via biolistic transfection in organotypic brain slices [21, 23]. However, brain injections in adults face challenges, such as potential interference with the tissue designated for acute slices, whereas the functional morphology of organotypic slices might not fully represent that of intact tissue. We, therefore, sought to explore viral transduction in vivo via neonatal intracerebroventricular (ICV) injections (Fig. 1a), aiming at efficient transgene expression in neurons, for up to 6 weeks post-infection for subsequent ex vivo imaging.

We employed the new generation of AAV-based sensor variant with a relatively high off-rate, AAV9.hSynap.iGluSnFR.WPRE.SV40, but also used the recently described low off-rate sensor variant, SF-iGluSnFR.A184S [24] for comparison. Although AAV9 appeared to penetrate more readily after ICV administration [25] than did AAV2/1, at three to 4 weeks post-injection, both methods provided efficient labelling of Schaffer collateral fragments in S. radiatum (Fig. 1c-e). The robust level of expression was maintained for at least 6 weeks post-infection, which made it suitable for ex vivo experiments in acute slices from young adult animals.

To validate the method, we set out to monitor iGluSnFR fluorescence intensity integrated across the region of interest (ROI, the area incorporating several axonal boutons) during electric stimulation of Schaffer collaterals (five stimuli $50 \mathrm{~ms}$ apart; imaging settings as described earlier [20]). For time-lapse imaging, we employed frame-scanning mode providing rapid sampling rate (pixel dwell time $0.5 \mu \mathrm{s}$, frame time $\sim 25 \mathrm{~ms}$ ) across the area of interest $(256 \times 96$ pixels, Fig. 1c). The recorded data sets were arranged as T-stacks, consisting of multiple frame scans (typically 35 to 50 , depending on the duration of recording). We thus achieved reliable imaging of the dynamics of glutamate release across the sampled tissue fragment (using a galvo mirror scanhead), with clear separation of five responses to individual electric stimuli (Fig. $1 \mathrm{c}$; fEPSP and $\Delta F / F_{O}$ signal traces; onetrial example). Our attempts to achieve a faster frame rate using a continuous resonant-scanner mode (with a Femtonics Femto-SMART scope) could not obtain a suitable trade-off between laser power and pixel dwell time to generate satisfactory signals without tissue damage, at least under the current protocol. Specific (non-continuous) regimes for resonant-scanner imaging may be required to achieve that.

A similar experiment using the slow-unbinding A184S sensor variant (Fig. 1d) revealed robust stimulus-evoked rises in the iGluSnFR intensity (Fig. 1e). However, this sensor variant did not seem to provide reliable separation between individual responses to five stimuli applied at $20 \mathrm{~Hz}$ (Fig. 1e; $\Delta F / F_{O}$ trace, one-trial example), thus pointing to the corresponding limitations in temporal resolution.

\section{Multi-synapse imaging of glutamate release at individual axonal boutons}

We next asked if the chosen frame-scanning method is sufficiently sensitive to document glutamate release at individual axonal boutons. We therefore used the recorded image-frame stacks to analyse fluorescence dynamics at small ROIs associated with individual axonal boutons (Fig. 2a). The fluorescence dynamics at individual selected boutons showed that recording sensitivity and signal-noise ratios were sufficient, in principle, to document individual glutamate releases (Fig. 2b; $\Delta F / F_{0}$ traces, four-trial average), at least in baseline conditions. For comparison purposes, we recorded a fragment of the same axon (as Fig. 2a) in linescan mode, which provides high temporal resolution $(\sim 1.45 \mathrm{~ms})$. The fluorescence dynamics thus recorded from three boutons of interest (Fig. 3a, bouton numbers as in Fig. 2a; one-trial example) was qualitatively similar to that obtained in the frame-scanning mode (compare boutons 5-7 in Fig. 2b and Fig. 3b).

\section{Imaging glutamate release during LTP induction}

One of the main advantages of the frame-scan mode (with galvo mirrors), as opposed to various linescan options, is relatively low overall (cumulative) laser exposure per pixel yet sufficient pixel dwell time to generate enough photons. Firstly, this lowers the propensity for irreversible photo-damage that may occur in cellular structures under intense laser light. Secondly, it reduces photobleaching of the fluorescent indicator, which has been a key prerequisite for stable longer-term imaging. As pointed out above, available parameters of the continuous resonant scanning fell outside the optimal range for the present protocol. 


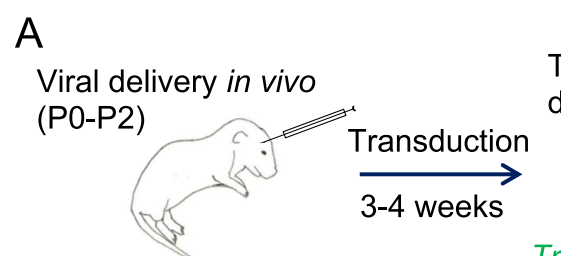

\section{[AAV9.hSynap.iGluSnFr]}

\section{Transduced \\ hippocampus}

dissection Acute slice $\lambda_{x}{ }^{2 P}=910 \mathrm{~nm}$

B

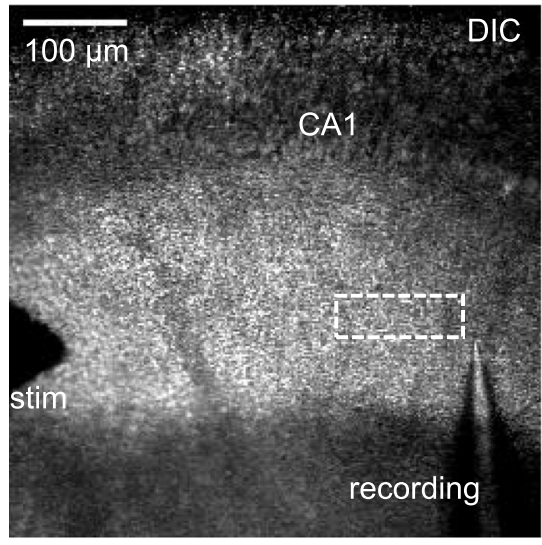

C
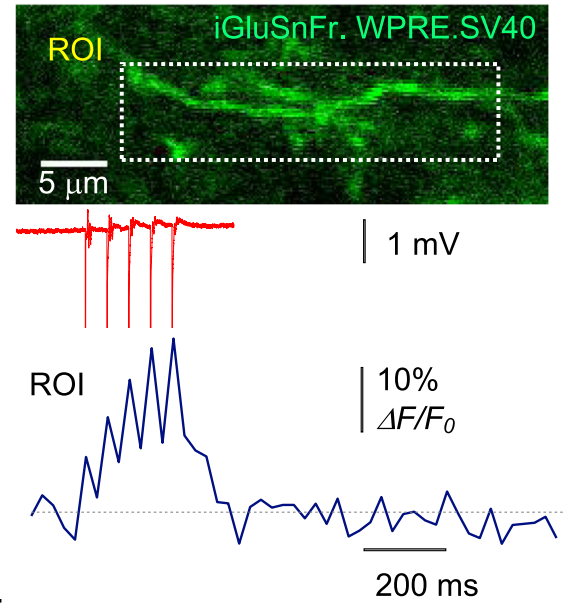

D

E
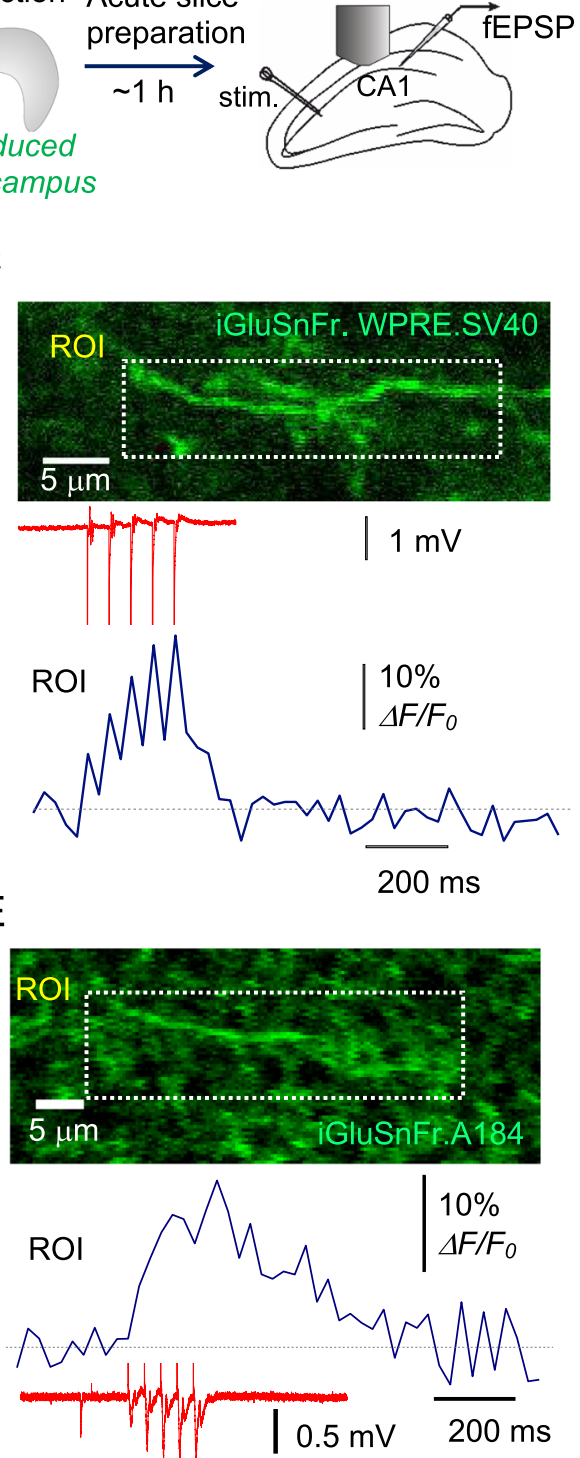

Fig. 1 Monitoring glutamate release from multiple axons ex vivo in hippocampal slices labelled with iGluSnFR through viral transduction in vivo. a A diagram depicting viral ICV injections in neonates (P0-P2) followed by AAV transduction (3-4 weeks), dissection of hippocampi, and acute slice preparation for two-photon excitation imaging coupled with electrophysiology (Schaffer collateral stimulation and fEPSP recording in $S$. radiatum). $\mathbf{b}$ Experimental arrangement as seen in the microscope (DIC channel); stimulating and recording electrodes are seen; dotted rectangle, ROI for imaging. c Image, axon fragment in S. radiatum (ROI as in B) as seen in the green channel (AAV9.hSynap.iGluSnFR.WPRE.SV40 fluorescence; 50-frame average). Upper trace, fEPSP response to afferent stimuli (five at $20 \mathrm{~Hz}$, one-trial example); lower trace, the corresponding ROI-averaged $\Delta F / F_{0}$ signal time course (one-trial example). d Arrangement as in (b) but for the 'slow-decay' sensor variant AAV2/1.hSyn.SF.iGluSnFR.A184S (green channel shown). e Experiment as in (c) but for AAV2/1.hSyn.SF.iGluSnFR.A184S; notation as in (c)

We therefore set out to explore our imaging method in an attempt to document changes, if any, of glutamate release during the high-frequency stimulation (HFS)-induced LTP. The classical LTP induction protocol in iGluSnFR-expressing acute slices produced a reliable increase in the fEPSP slope, lasting for up to $90 \mathrm{~min}$ postinduction (example in Fig. 4a). In selected areas of $S$. radiatum, we thus identified groups of candidate axonal boutons that responded to afferent stimulation but also remained firmly in focus during the experiment, to reduce any bias associated with focal drift (Fig. 4b). The boutons selected based on this mandatory criterion, were not necessarily the boutons showing the best signal-to-noise ratios of their $\Delta F / F_{O}$ responses (this may 


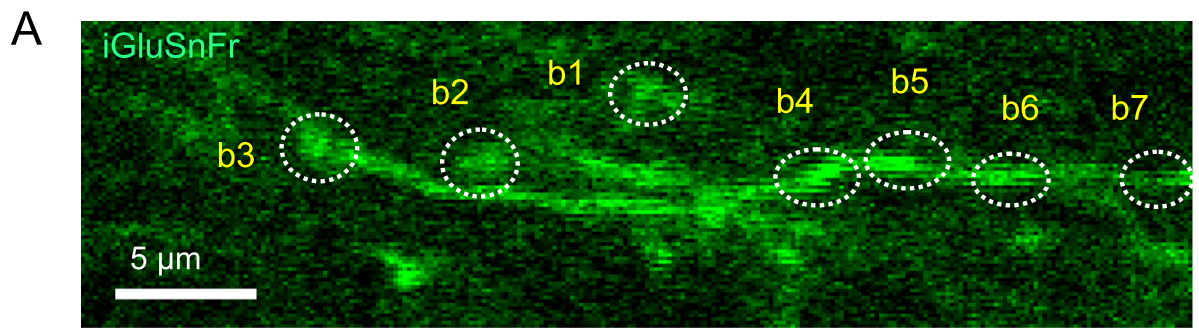

B

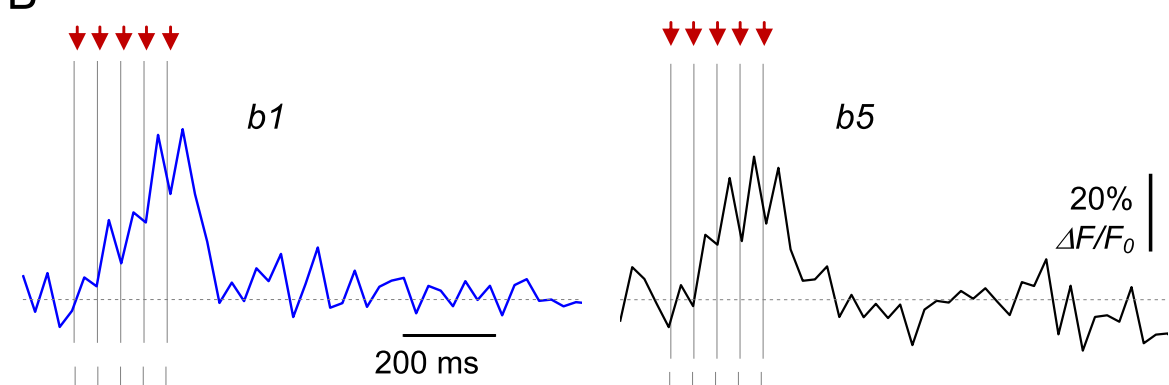

b2

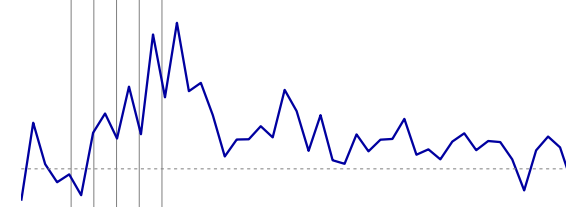

b6
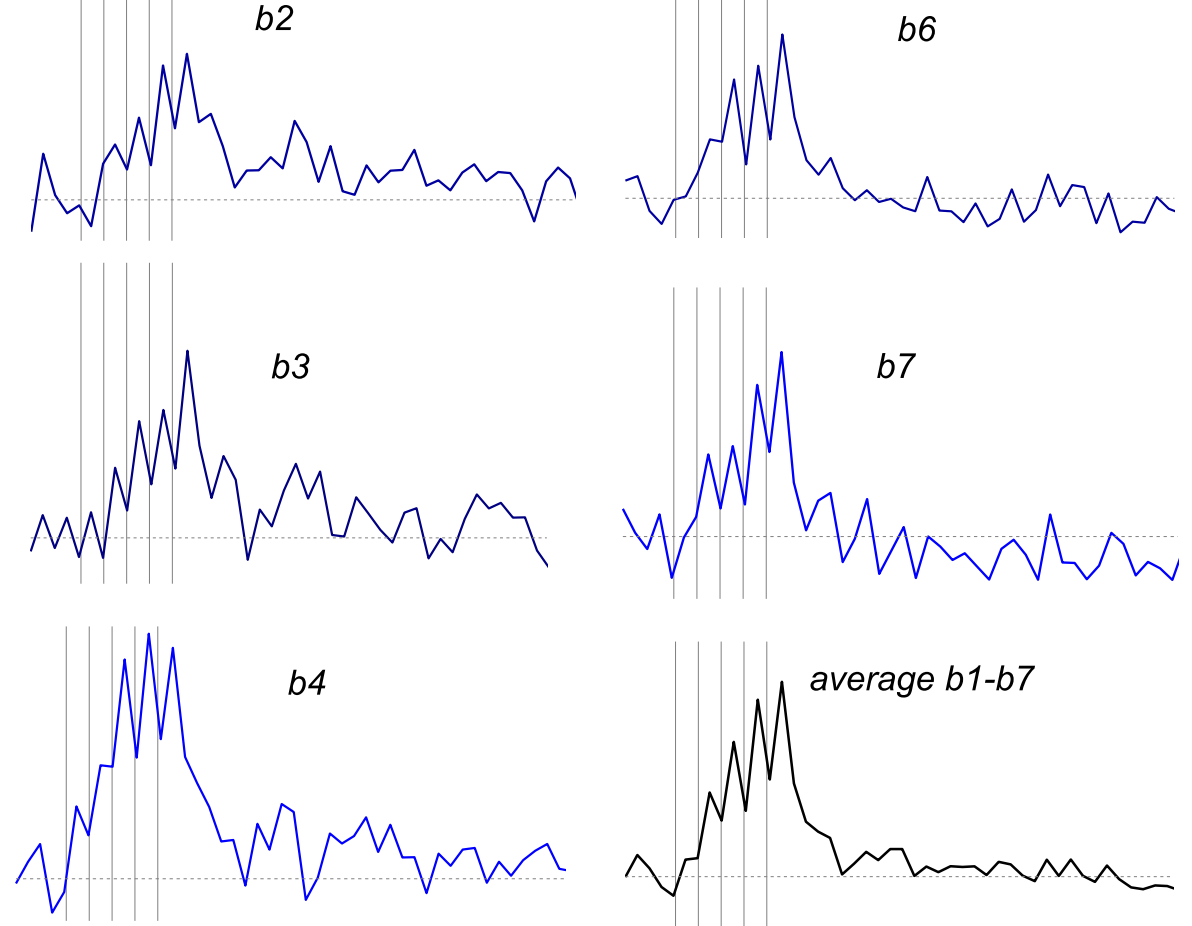

Fig. 2 Optical monitoring of glutamate release from multiple synapses using the AAV9.hSynap.iGluSnFR.WPRE.SV40 imaging in fast frame-scan mode. a Axonal fragments in S. radiatum (same region as in Fig. 1c), showing candidate presynaptic boutons (b1-b8). Image is average of 50 frames of the T-stack. $\mathbf{b}$ Traces, $\Delta F / F_{0}$ signal time course within individual ROls that correspond to boutons b1-b7 shown in (a) and the b1-b7 average trace, as indicated, during afferent stimulation (five pulses at $20 \mathrm{~Hz}$; four-trial average)

also relate to varied iGluSnFR expression). Whilst individual boutons displayed varied effects of LTP induction on the fluorescence dynamics of iGluSnFR, they nonetheless appeared to indicate a clear trend towards an increase in the $\Delta F / F_{O}$ signal amplitude (example in Fig. 4c).
This trend was more prominent when the areaintegrated $\Delta F / F_{O}$ signals (as in Fig. 1c, e) were compared (Fig. 5a). To evaluate this quantitatively, we first measured the iGluSnFR signal amplitude $\left\{\Delta F / F_{0}\right\}$, the mean $\Delta F / F_{O}$ value measured over $300 \mathrm{~ms}$ after the first stimulus onset (Fig. 5a, traces), 1-5 min prior to LTP induction, and 30 


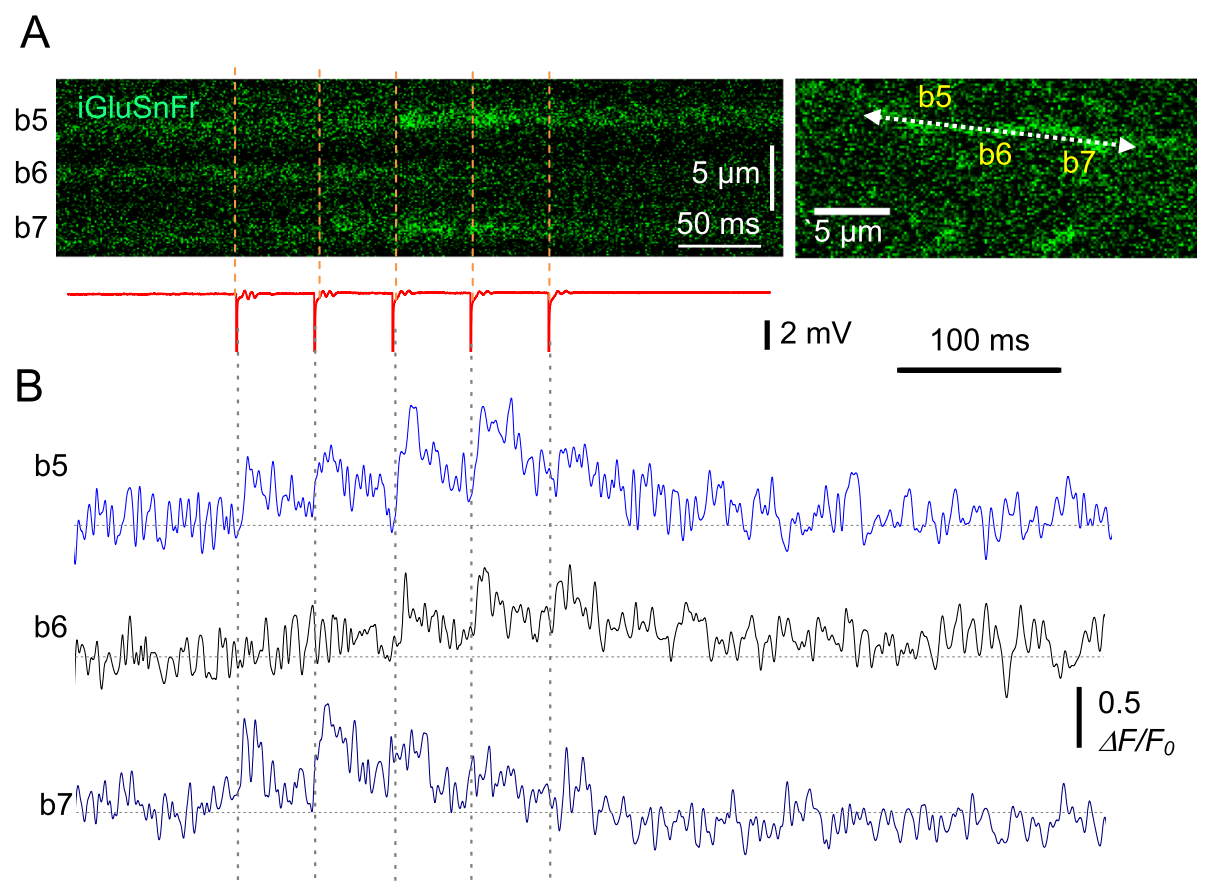

Fig. 3 Documenting glutamate release from multiple axonal boutons using linescan imaging mode. a Linescan image (left) depicting the iGluSnFR.WPRE.SV40 fluorescence time course in three axonal boutons (b5-b7, right; ROI as in Fig. 2) during afferent stimulation (five pulses at 20 $\mathrm{Hz}$ ), with fEPSP monitoring (red trace). b Traces, $\Delta F / F_{0}$ signal time course recorded as shown in (a) (one-trial example).

min and 90 min after LTP induction. Comparing these $\left\{\Delta F / F_{0}\right\}$ values within individual slices revealed a significant increase after LTP induction (from $3.1 \pm 0.9 \%$ to $7.2 \pm 2.2 \%$ at $30 \mathrm{~min}$ post-HFS, $p<0.035$; to $6.5 \pm 1.6 \%$ at 90 min post-HFS, $p<0.005 ; n=7$ slices, paired $t$-test). Second, we compared full $\Delta F / F$ responses at the same time points. To achieve paired comparison, we normalised post-HFS traces by the $\left\{\Delta F / F_{0}\right\}$ value of the pre-LTP response, within each individual preparation (slice), and then re-scaled all the traces to match the sample-average $\left\{\Delta F / F_{0}\right\}$ value in baseline conditions (Fig. 5c). Again, this paired-comparison design revealed a prominent increase in the $\Delta F / F_{O}$ signal at 30 and $90 \mathrm{~min}$ after LTP induction (Fig. 5c). Whether such an increase necessarily indicates a greater amount of evoked glutamate release is discussed below.

\section{Blocking astroglial glutamate transport saturates iGluSnFR signal}

Because the $\Delta F / F_{O}$ signal we record reflects glutamate binding to iGluSnFR molecules, it may compete with other (invisible) binding sites for glutamate in the neuropil. It has been well established that, once released from presynaptic boutons in the hippocampus, $>90 \%$ of glutamate molecules are bound and taken up by highaffinity astroglial glutamate transporters [26]. These transporters will therefore compete with iGluSnFR for glutamate binding and removal from the extracellular space, prompting a hypothesis that their inhibition could boost the iGluSnFR signal. To test this, we added the transporter blocker TFB-TBOA to the bath $(50 \mu \mathrm{M})$, after recording a reliable $\Delta F / F_{O}$ response $90 \mathrm{~min}$ after LTP induction. Within $3 \mathrm{~min}$ after TBOA application, afferent stimulation induced a large, virtually irreversible increase in the iGluSnFR $\Delta F / F_{O}$ signal (Fig. $5 \mathrm{~d}$ ). The signal has become undetectable within the next few minutes, most likely due to the progressive saturation of iGluSnFR by the excess of extracellular glutamate in TBOA (Fig. 5d). At the same time, TBOA had little effect on the fast fEPSPs (Fig. 5d, fEPSP traces), reflecting no detectable influence on glutamate release, in line with earlier reports [27, 28]. These results indicate that the LTP-associated increase in the iGluSnFR $\Delta F / F_{O}$ signal could potentially be related to the reduced presence of astroglial glutamate transporters in the perisynaptic environment.

\section{Discussion}

In this study, we took advantage of the recently developed, genetically encoded optical iGluSnFR sensors for glutamate $[19,24]$, in an attempt to detect changes in glutamate release following the induction of LTP. We have successfully transduced the sensors in hippocampal Schaffer collateral fibres using neonatal viral infection. We explored the suitability of the fast frame-scanning (two-photon excitation) imaging mode for monitoring optical glutamate 


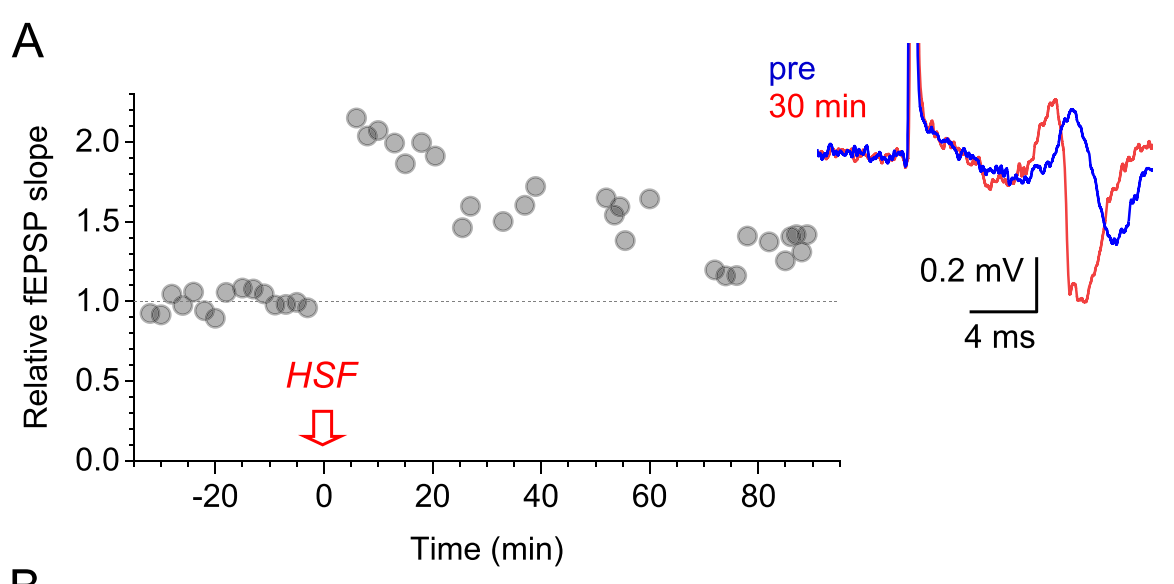

B
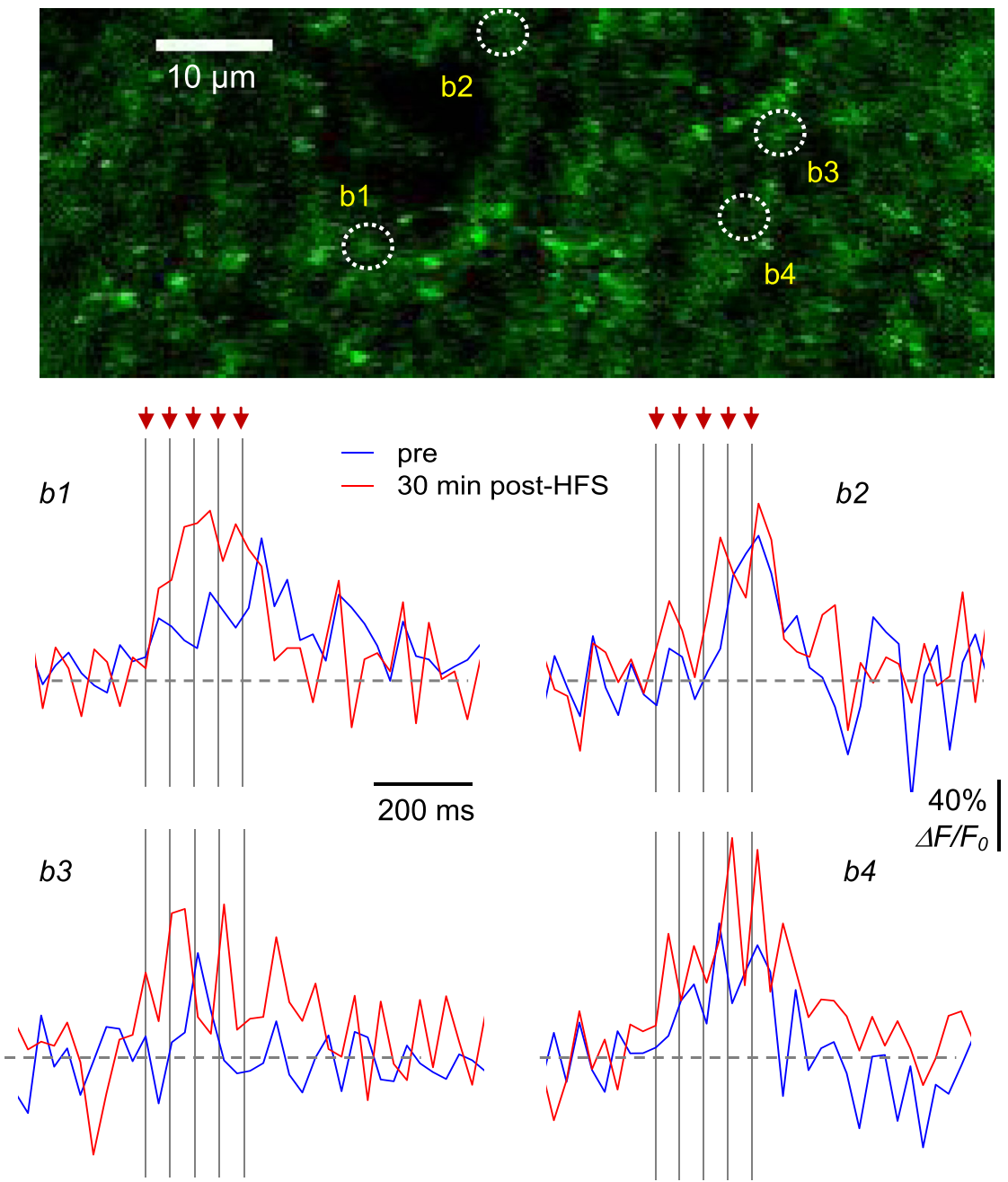

Fig. 4 Optical glutamate signal at individual axonal boutons during LTP induction. a Characteristic time course of the fEPSP slope recorded in S. radiatum following LTP induction by high frequency stimulation (HFS, one-slice example). Traces, the corresponding fEPSP examples in baseline conditions (blue) and 30 min after LTP induction (red). b Image, ROI in S. radiatum (iGluSnFR.WPRE.SV40 channel) showing 4 axonal boutons, b1-b4, designated for glutamate release monitoring. Traces, iGluSnFR $\Delta F / F_{0}$ signal recorded from boutons b1-b4 before (blue) and $\sim 30 \mathrm{~min}$ after (red) LTP induction. Traces are single-trial examples; arrows and dotted lines, afferent stimulus timestamps 

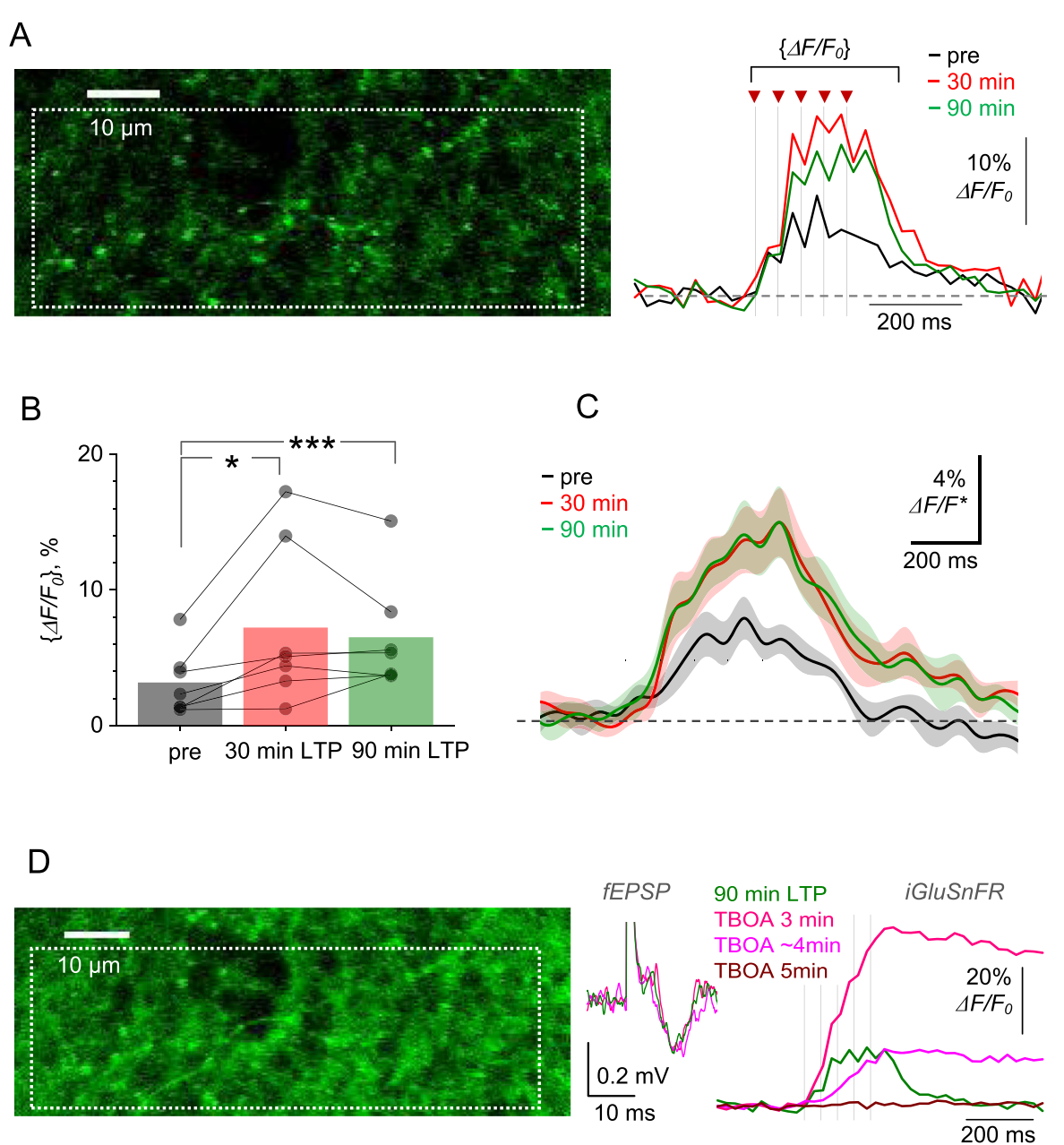

Fig. 5 LTP induction at CA3-CA1 synapses boosts optical glutamate signal in the S. radiatum neuropil. a Image, axon fragment in S. radiatum showing the area with multiple axonal boutons (dotted rectangle, iGluSnFR.WPRE.SV40 channel) for the analysis of average iGluSnFR $\Delta F / F_{0}$ signal (right traces), as shown before (pre), 30 min after (red), and 90 min after HFS. One-slice example; traces, singletrial examples; arrows and dotted lines, afferent stimulus timestamps. Averaging interval for calculating $\left\{\Delta F / F_{0}\right\}$ values is shown. b ROl-average iGluSnFR $\left\{\Delta F / F_{0}\right\}$ values in baseline conditions (pre), and at $30 \mathrm{~min}$ and $90 \mathrm{~min}$ after LTP induction, as indicated. Connected dots, individual slice data; bars, average values $(n=7)$. ${ }^{*} p<0.04 ;{ }^{* * *} p<0.005$. c Average iGluSnFR $\Delta F / F_{0}$ signal traces (line \pm shaded area, mean $\pm S E M, n=7$ ) normalised to their $\left\{\Delta F / F_{0}\right\}$ value in baseline conditions, in each individual preparation, and rescaled to illustrate the 'average $\Delta F / F_{0}$ traces' across preparations $\left(\Delta F / F^{*}\right)$. $\mathbf{d}$ Experiment as in $(\mathbf{a})$ but following the blockade of glutamate transporters with $50 \mu \mathrm{M}$ TBOA, at 90 min after LTP induction. fEPSP and iGluSnFR traces illustrate single trials recorded at different time points after TBOA application onset, as indicated; one-slice example, notations as in (a). Note that no $\Delta F / F_{0}$ signal (red) may reflect saturation of the baseline fluorescence $F_{0}$

signals from multiple axonal boutons, thus identifying some advantages and limitations, in the context. The key advantage rests with the reduced exposure to laser light and the ability to record from multiple synaptic connections, in some cases with satisfactory sensitivity and temporal resolution. This is, however, somewhat offset by the fact that during longer-term recordings and / or intense electric stimulation the tissue is likely to drift while also altering its morphological features, albeit on the microscopic scale. Because such movements could alter geometry and position of the fluorescence source(s), this could potentially bias the readout of dynamic optical recordings. Selecting the objects of interest, such as axonal boutons, based on their morphological stability, potentially leads to suboptimal sampling in terms of the signalto-noise ratio. The future improvements of the technique could combine a better controlled labelling of axons, aiming at a sufficiently high level of iGluSnFR expression within targeted sub-microscopic structures. Improved out-of-focus detection could be achieved by using the vertically extended point-spread function of the two-photon excitation system [29], which is sometimes termed 'Bessel beam'. The latter should help overcome the effects of 3D drift, and therefore improve the sampling procedure. 
Notwithstanding its potential limitations, another advantage of the present approach is its unbiased way of sampling axonal boutons. As the majority of excitatory synapses, or at least a significant proportion of them, are of low release probability [21, 30], one would expect boutons that are sampled in an unbiased way to show a relatively low glutamate signal on average, as we find here. It is likely that in other data sets, in which boutons are selected based on a high signal to noise ratio, represent higher release probability synapses.

The present approach has identified a significant increase in the average optical glutamate signal in the Schaffer collateral neuropil, up to $\sim 90 \mathrm{~min}$ after LTP induction at CA3-CA1 synapses. This increase is unlikely to reflect the 'transient-LTP' component, which is expressed presynaptically but decays within 70-80 afferent discharges [31], or within 15-20 min under the present protocol. At first glance, the increase in iGluSnFR fluorescence must indicate an increased amount of released glutamate in response to afferent stimulation. However, the fluorescent signal of iGluSnFR reports glutamate molecules bound to the indicator. Any endogenous high-affinity glutamate buffer that competes with this binding process can potentially affect optical readout. Intriguingly, hippocampal neuropil is equipped with such a buffer, in the shape of high-affinity glutamate transporters that are expressed, at high density, on the surface of astroglia [26, 32, 33]. Thus, a significant decrease in the numbers of locally available glutamate transporters could boost glutamate binding to its optical sensor upon evoked release. Indeed, when we blocked astroglial glutamate transporters with TBOA, the iGluSnFR signal was first boosted then entirely saturated, reflecting an excess of extracellular glutamate. At the same time, no increase in glutamate release efficacy could be detected. Whether a similar mechanism is enacted during LTP induction is an intriguing and important question yet to be fully addressed.

On a more general note, the remaining uncertainty about release probability changes during LTP is unlikely to be resolved unambiguously without considering the effects of LTP induction on both potentiated and (neighbouring) non-potentiated synapses, and possibly on the local astroglial microenvironment. Similarly, it would seem important to employ an unbiased sampling method that would include all activated synapses (e.g., represented by axonal boutons or dendritic spines), regardless of their baseline synaptic efficacy or signal detectability.

\section{Methods}

\section{Viral transduction for labelling axonal boutons within CA3-CA1 region}

All animal procedures were conducted in accordance with the European Commission Directive (86/609/ EEC), the United Kingdom Home Office (Scientific Procedures) Act
(1986). For the experiments, both male and female C57BL/6 J mice (Charles River Laboratories) were used. For ex vivo imaging of individual boutons, an AAV virus expressing the neuronal optical glutamate sensor, AAV9.hSynap.iGluSnFR.WPRE.SV40, supplied by Penn Vector Core (PA, USA) was injected into the cerebral ventricles of neonates. For viral gene delivery, pups, male and female (P0-P1), were prepared for aseptic surgery. To ensure proper delivery, intracerebroventricular (ICV) injections were carried out after a sufficient visualization of the targeted area [34]. Viral particles were injected in a volume $2 \mu \mathrm{l} /$ hemisphere (totalling $5 \times 10^{9}$ genomic copies), using a glass Hamilton microsyringe at a rate not exceeding of $0.2 \mu \mathrm{l} / \mathrm{s}, 2 \mathrm{~mm}$ deep, perpendicular to the skull surface, guided to a location approximately $0.25 \mathrm{~mm}$ lateral to the sagittal suture and $0.50-0.75 \mathrm{~mm}$ rostral to the neonatal coronary suture. Once delivery was completed, the microsyringe was left in place for $20-30 \mathrm{~s}$ before being retracted. Pups (while away from mothers) were continuously maintained in a warm environment to eliminate risk of hypothermia in neonates. After animals received AAV injections, they were returned to the mother in their home cage. Pups were systematically kept as a group of litters. Every animal was closely monitored for signs of hypothermia following the procedure and for days thereafter, to ensure that no detrimental side effects appear. For transduction of glutamate sensors in vivo, there were three- to four- weeks to suffice.

\section{Preparation of acute hippocampal slices}

Acute hippocampal slices $(350 \mu \mathrm{m}$ thick) were prepared from three- to 4 week-old mice. The hippocampal tissue was sliced in an ice-cold slicing solution containing (in $\mathrm{mM}): 64 \mathrm{NaCl}, 2.5 \mathrm{KCl}, 1.25 \mathrm{NaH}_{2} \mathrm{PO}_{4}, 0.5 \mathrm{CaCl}_{2}, 7$ $\mathrm{MgCl}_{2}, 25 \mathrm{NaHCO}_{3}, 10 \mathrm{D}$-glucose and 120 sucrose, saturated with $95 \% \mathrm{O}_{2}$ and $5 \% \mathrm{CO}_{2}$. Acute slices were then transferred into a bicarbonate-buffered Ringer solution containing (in mM) $126 \mathrm{NaCl}, 3 \mathrm{KCl}, 1.25 \mathrm{NaH}_{2} \mathrm{PO}_{4}, 2$ $\mathrm{MgSO}_{4}, 2 \mathrm{CaCl}_{2}, 26 \mathrm{NaHCO}_{3}, 10 \mathrm{D}$-glucose continuously bubbled with $95 \% \mathrm{O}_{2}$ and $5 \% \mathrm{CO}_{2}$ (pH 7.4; 300$310 \mathrm{mOsmol}$ ). Slices were allowed to rest for at least 60 min before the recordings started. For recordings, slices were transferred to a recording chamber mounted on the stage of an Olympus BX51WI upright microscope (Olympus, Tokyo, Japan) and superfused at $31-33^{\circ} \mathrm{C}$.

\section{Two-photon (2P) excitation fluorescent imaging of glutamate release}

2P excitation microscopy was carried out using an Olympus FV10MP imaging system optically linked to a Ti:Sapphire MaiTai femtosecond-pulse laser (SpectraPhysics-Newport), equipped with galvo scanners, and integrated with patch-clamp electrophysiology. Acute hippocampal slices were illuminated at $\lambda_{\mathrm{x}}{ }^{2 \mathrm{P}}=910 \mathrm{~nm}$ 
(iGluSnFR optimum) in the green emission channel. In s.radiatum of area CA1, we focused on axonal fragments that (a) were expressing the optical sensor at a level sufficient to visualise individual axonal boutons, and (b) responded to electric stimulation of Schaffer collaterals with the iGluSnFR signal rise. For time-lapse imaging of the iGluSnFR signal (before, during, and after evoked glutamate release), images were collected in frame scan mode to provide fast acquisition rates and a short pixel dwell time. Frame scans were acquired with a pixel dwell time of $0.5 \mu \mathrm{s}$, at a nominal resolution of $\sim 5-7$ pixels per $\mu \mathrm{m}(256 \times 96)$. To minimize photodamage, only a single focal section through the region of interest (ROI) containing selected axonal fragments was acquired, at a relatively low laser power (3-6 $\mathrm{mW}$ under the objective). The focal plane was regularly adjusted, to account for specimen drift. Time-lapse frame scans of ROIs (containing multiple boutons within the focal plane) were acquired before and up to 60-90 min after the induction of LTP, as detailed below.

The optical signal of the iGluSnFR was expressed as the $\left(F(t)-F_{0}\right) / F_{0}=\Delta F / F_{0}$, where $F(t)$ stands for intensity over time, and $F_{0}$ is the baseline intensity averaged over $\sim 150 \mathrm{~ms}$ prior to the stimulus. To quantify LTPinduced changes in the average optical glutamate signal, we calculated the $\left\{\Delta F / F_{0}\right\}$ value representing the mean $\Delta F / F_{0}$ signal over the $300 \mathrm{~ms}$ interval from the first stimulus onset.

\section{Electrophysiology ex vivo: LTP induction}

Glutamate release was evoked by stimulation of the bulk of Schaffer collaterals, using a concentric bipolar electrode $(100 \mu \mathrm{s}, 20-200 \mu \mathrm{A}$; corresponding to approximately one third of the saturating reponse) placed in the $S$. radiatum. Evoked field excitatory postsynaptic potentials (fEPSPs) were monitored with an extracellular recording pipette positioned in S. radiatum $>200 \mu \mathrm{m}$ away from the stimulating electrode. The recording electrode has a resistance of 1.5-2 M $\Omega$ when filled with a Ringer solution. fEPSPs were recorded using a Multipatch 700B amplifier controlled by the pClamp 10.2 software (Molecular Devices, USA).

Synaptic responses were evoked by a brief burst of stimuli consisting of five pulses applied at $20 \mathrm{~Hz}, 50 \mathrm{~ms}$ apart. Basal synaptic transmission was monitored for 10 to $20 \mathrm{~min}$ (every $30 \mathrm{~s}$ to $1 \mathrm{~min}, \sim 0.03 \mathrm{~Hz}$ ) before implementing a high-frequency stimulation (HFS) protocol for the induction of LTP. The HFS protocol contained of three trains of stimuli $(100$ pulses at $100 \mathrm{~Hz})$, applied in a 60-s interval. GABA receptors were blocked with $100 \mu \mathrm{M}$ picrotoxin and $3 \mu \mathrm{M}$ CGP-52432 (in bath). The fEPSP slope was typically monitored for at least an hour (up to $2 \mathrm{~h}$ ) post-HFS, using the same stimulation protocol (five pulses at $20 \mathrm{~Hz}$ ).

\section{Statistical analyses}

All data are presented as mean \pm standard error of the mean (SEM), with $n$ referring to the number of slices analysed. For the statistical difference between baseline and two time points after LTP conditions, paired-sample comparison (paired-sample $t$-test) was performed for the $\left\{\Delta F / F_{0}\right\}$ values, as described.

\section{Acknowledgements \\ The authors thank Loren Looger and Jonathan Marvin for providing original variants of iGluSnFR.}

\section{Authors' contributions}

OK carried out experimental studies, analysed the results, compiled

illustrations, and contributed to manuscript writing; KZ implemented optical designs and image analyses; DAR narrated the study, analysed selected data, and wrote the manuscript. The authors read and approved the final manuscript.

\section{Funding}

This study was supported by the Wellcome Trust Principal Fellowship (212251_Z_18_Z), ERC Advanced Grant (323113) and European Commission NEUROTWIN grant (857562), to D.A.R.

Availability of data and materials

The datasets obtained and/or analysed during the current study are available from the corresponding author on reasonable request.

Ethics approval and consent to participate

Not applicable.

Consent for publication

Not applicable.

\section{Competing interests}

The authors declare that they have no competing interests.

Received: 17 December 2019 Accepted: 27 February 2020

Published online: 13 March 2020

\section{References}

1. Hebb DO. The Organization of Behavior. New York: Wiley; 1949.

2. Bliss T, Collingridge G. A synaptic model of memory - long-term potentiation in the Hippocampus. Nature. 1993;361(6407):31-9.

3. Bliss T, Lomo T. Long-lasting potentiation of synaptic transmission in the dentate area of the anaesthetized rabbit following stimulation of the perforant path. J Physiol. 1973;232(2):331-56.

4. Nicoll RA. A brief history of long-term potentiation. Neuron. 2017;93(2): 281-90.

5. Huang YY, Zakharenko SS, Schoch S, Kaeser PS, Janz R, Sudhof TC, et al. Genetic evidence for a protein-kinase-A-mediated presynaptic component in NMDA-receptor-dependent forms of long-term synaptic potentiation. Proc Natl Acad Sci U S A. 2005;102(26):9365-70.

6. Malgaroli A, Ting AE, Wendland B, Bergamaschi A, Villa A, Tsien RW, et al. Presynaptic component of long-term potentiation visualized at individual hippocampal synapses. Science. 1995;268(5217):1624-8.

7. Emptage NJ, Reid CA, Fine A, Bliss TV. Optical quantal analysis reveals a presynaptic component of LTP at hippocampal Schaffer-associational synapses. Neuron. 2003;38(5):797-804

8. Kullmann DM. Amplitude fluctuations of dual-component Epscs in hippocampal pyramidal cells - implications for long-term potentiation. Neuron. 1994;12(5):1111-20.

9. Isaac JT, Nicoll RA, Malenka RC. Evidence for silent synapses: implications for the expression of LTP. Neuron. 1995;15(2):427-34.

10. Diamond JS, Bergles DE, Jahr CE. Glutamate release monitored with astrocyte transporter currents during LTP. Neuron. 1998;21(2):425-33.

11. Luscher C, Malenka RC, Nicoll RA. Monitoring glutamate release during LTP with glial transporter currents. Neuron. 1998;21(2):435-41. 
12. Ward B, McGuinness L, Akerman CJ, Fine A, Bliss TV, Emptage NJ. Statedependent mechanisms of LTP expression revealed by optical quantal analysis. Neuron. 2006;52(4):649-61.

13. Enoki R, Hu YL, Hamilton D, Fine A. Expression of long-term plasticity at individual synapses in hippocampus is graded, bidirectional, and mainly presynaptic: optical quantal analysis. Neuron. 2009;62(2):242-53.

14. Lynch GS, Dunwiddie T, Gribkoff V. Heterosynaptic depression: a postsynaptic correlate of long-term potentiation. Nature. 1977;266(5604): 737-9.

15. Ibata K, Sun Q, Turrigiano GG. Rapid synaptic scaling induced by changes in postsynaptic firing. Neuron. 2008;57(6):819-26.

16. Delvendahl I, Kita K, Muller M. Rapid and sustained homeostatic control of presynaptic exocytosis at a central synapse. Proc Natl Acad Sci U S A. 2019;116(47):23783-9.

17. Ryan TA, Reuter H, Smith SJ. Optical detection of a quantal presynaptic membrane turnover. Nature. 1997;388(6641):478-82.

18. Sankaranarayanan S, Ryan TA. Real-time measurements of vesicle-SNARE recycling in synapses of the central nervous system. Nat Cell Biol. 2000;2(4):197-204

19. Marvin JS, Borghuis BG, Tian L, Cichon J, Harnett MT, Akerboom J, et al. An optimized fluorescent probe for visualizing glutamate neurotransmission. Nat Methods. 2013;10(2):162-70.

20. Jensen TP, Zheng K, Tyurikova O, Reynolds JP, Rusakov DA. Monitoring single-synapse glutamate release and presynaptic calcium concentration in organised brain tissue. Cell Calcium. 2017;64:102-8.

21. Jensen TP, Zheng KY, Cole N, Marvin JS, Looger LL, Rusakov DA. Multiplex imaging relates quantal glutamate release to presynaptic $\mathrm{Ca} 2+$ homeostasis at multiple synapses in situ. Nat Commun. 2019;10:1.

22. Reynolds JP, Zheng K, Rusakov DA. Multiplexed calcium imaging of singlesynapse activity and astroglial responses in the intact brain. Neurosci Lett. 2018;689:26.

23. Jensen TP, Zheng K, Cole N, Marvin JS, Looger LL, Rusakov DA. Multiplex imaging relates quantal glutamate release to presynaptic $\mathrm{Ca}(2+)$ homeostasis at multiple synapses in situ. Nat Commun. 2019;10(1):1414.

24. Marvin JS, Scholl B, Wilson DE, Podgorski K, Kazemipour A, Muller JA, et al. Stability, affinity, and chromatic variants of the glutamate sensor iGluSnFR. Nat Methods. 2018;15(11):936-9.

25. Hammond SL, Leek AN, Richman EH, Tjalkens RB. Cellular selectivity of AAV serotypes for gene delivery in neurons and astrocytes by neonatal intracerebroventricular injection. PLoS One. 2017;12(12):e0188830.

26. Danbolt NC. Glutamate uptake. Prog Neurobiol. 2001;65:1-105.

27. Zheng K, Scimemi A, Rusakov DA. Receptor actions of synaptically released glutamate: the role of transporters on the scale from nanometers to microns. Biophys J. 2008;95(10):4584-96.

28. Chamberland S, Evstratova A, Toth K. Interplay between synchronization of multivesicular release and recruitment of additional release sites support short-term facilitation at hippocampal mossy fiber to CA3 pyramidal cells synapses. J Neurosci. 2014;34(33):11032-47.

29. Scott R, Rusakov DA. Main determinants of presynaptic $\mathrm{Ca}^{2+}$ dynamics at individual mossy fiber-CA3 pyramidal cell synapses. J Neurosci. 2006;26(26): 7071-81.

30. Murthy VN, Sejnowski TJ, Stevens CF. Heterogeneous release properties of visualized individual hippocampal synapses. Neuron. 1997;18(4):599-612.

31. Volianskis A, Jensen MS. Transient and sustained types of long-term potentiation in the CA1 area of the rat hippocampus. J Physiol. 2003;550(2): 459-92.

32. Diamond JS, Jahr CE. Transporters buffer synaptically released glutamate on a submillisecond time scale. J Neurosci. 1997;17(12):4672-87.

33. Danbolt NC, Chaudhry FA, Dehnes Y, Lehre KP, Levy LM, Ullensvang K, et al. Properties and localization of glutamate transporters. Prog Brain Res. 1998:116:23-43.

34. Kim JY, Ash RT, Ceballos-Diaz C, Levites Y, Golde TE, Smirnakis SM, et al. Viral transduction of the neonatal brain delivers controllable genetic mosaicism for visualising and manipulating neuronal circuits in vivo. Eur J Neurosci. 2013;37(8):1203-20.

\section{Publisher's Note}

Springer Nature remains neutral with regard to jurisdictional claims in published maps and institutional affiliations.

Ready to submit your research? Choose BMC and benefit from:

- fast, convenient online submission

- thorough peer review by experienced researchers in your field

- rapid publication on acceptance

- support for research data, including large and complex data types

- gold Open Access which fosters wider collaboration and increased citations

- maximum visibility for your research: over $100 \mathrm{M}$ website views per year

At BMC, research is always in progress.

Learn more biomedcentral.com/submissions 